\title{
Novel Analytical and Numerical Methods in Heat Transfer Enhancement and Thermal Management
}

\author{
Assunta Andreozzi, ${ }^{1}$ Guy Lauriat, ${ }^{2}$ Qiuwang Wang, ${ }^{3}$ Sotirios Karellas, ${ }^{4}$ and Yogesh Jaluria ${ }^{5}$ \\ ${ }^{1}$ Dipartimento di Ingegneria Industriale, Università degli Studi di Napoli Federico II, 80125 Napoli, Italy \\ ${ }^{2}$ Laboratoire de Modélisation et Simulation Multi Echelle, Equipe Transferts de Chaleur et de Matière, Université PARIS-EST, \\ 77454 Marne-la-Vallée Cedex 2, France \\ ${ }^{3}$ School of Energy and Power Engineering, Xian Jiaotong University, Xian, Shaanxi 710049, China \\ ${ }^{4}$ Laboratory of Steam Boilers and Thermal Plants, School of Mechanical Engineering, National Technical University of Athens, \\ Zografou, 15780 Athens, Greece \\ ${ }^{5}$ Mechanical and Aerospace Engineering Department, Rutgers, the State University of New Jersey, Piscataway, NJ 08854-8058, USA
}

Correspondence should be addressed to Assunta Andreozzi; assunta.andreozzi@unina.it

Received 5 November 2015; Accepted 5 November 2015

Copyright (C) 2016 Assunta Andreozzi et al. This is an open access article distributed under the Creative Commons Attribution License, which permits unrestricted use, distribution, and reproduction in any medium, provided the original work is properly cited.

Heat transfer enhancement (HTE) and thermal management (THEMA) are very attractive issues in the research and industry fields. They play an important role in improving energy efficiency and developing high performance thermal systems. HTE and THEMA techniques are powerful tools to increase and improve heat transfer rate and thermal performance as well as to reduce the size of heat transfer systems in installation and operational costs.

The basic purpose of this special issue is to collect original research articles on the most recent analytical and numerical models applied in this field, with the purpose of providing guidelines for future research direction.

This special issue showed novel and interesting analytical and numerical procedures applied in heat transfer enhancement and thermal management.

The topics of the accepted papers cover a wide area of the heat transfer field, from the transient heat transfer to the heat transfer in porous media and to the system design and optimization in forced convection.

A brief overview of each manuscript selected for this special issue is presented in the following, in alphabetical order of the first author.

C. Devaraj et al. investigate numerically natural convection heat transfer in a two-dimensional square enclosure at various angles of inclination using a finite volume based computational procedure. The heat transfer is from a constant temperature heat source of finite length centered at one of the walls to the cold wall on the opposite side while the remaining walls are insulated. The authors analyze the effect of area ratio of the heat source, Rayleigh number, and angle of inclination of the enclosure on the flow field and the heat transfer characteristics. Moreover the paper shows an exhaustive verification and validation section and gives heat transfer correlation equations for each angle of inclination of enclosure investigated which are in good agreement with the numerical results.

I. Simões et al. present, in their paper, a set of fully analytical solutions, together with explicit expressions, in the time and frequency domain for the heat conduction response of homogeneous unbounded and of bounded rectangular spaces (three-, two-, and one-dimensional spaces) subjected to point, line, and plane heat diffusion sources. The authors pay particular attention to the case of spatially sinusoidal, harmonic line sources. This last solution, referred to in the literature as the $2.5 \mathrm{D}$ problem. Proposed Green's functions are combined using an image source technique to model a half space, a corner, a layer system, a laterally confined layer system, a solid rectangular column, a solid rectangular column with an end cross section, and a 3D parallelepiped inclusion. This is the first such derivation that is expected 
to be efficient for formulating 3D thermodynamics problems using boundary elements and integral transforms. The solutions are validated by comparing computed results with those obtained directly in the time domain.

T.-W. Tu and S.-Y. Lee develop for the first time an analytical solution for the heat transfer in hollow cylinders with time-dependent boundary condition and timedependent heat transfer coefficient at different surfaces. The methodology employed by the authors is an extension of the shifting function method and permits transforming the system into a partial differential equation with homogenous boundary conditions. The transformed system is thus solved by series expansion theorem. Limiting cases of the solution are studied and numerical results are compared with those in the literature. The authors investigate the influence of physical parameters on the temperature distribution of a hollow cylinder along the radial direction, too.

$\mathrm{X}$. Zhang and R. Li present two papers. In the first one they investigate the cooling and radiation of a vectoring nozzle and compute the gas spectral characteristic in infrared band, adopting a narrow band model. The radiative heat transfer between the hot gas and the wall is considered with an enclosure model whereas the calculation of film cooling is performed through a cooling effectiveness method. A coupled heat balance equation of heat flux and wall temperature is established on the multilayer structure of the nozzle, including the wall, heat shield, and outer shield, and a Newton-Raphson scheme is taken for solution. The results verification is carried out by means of the investigation of temperature on the expansion part of an experimental nozzle in NASA TN D-1988. The authors also investigate another vectoring nozzle with a multirow of film cooling.

The same authors investigate the similarity of plume radiation between reduced scaling solid rocket models and full scale ones in ground. In the second paper the authors compute flow and radiation of plume from solid rockets with scaling ratio from 0.1 to 1 , using CFD code. The radiative transfer equation is solved by the finite volume method in the infrared band $2 \sim 6 \mu \mathrm{m}$. The spectral characteristics of plume gases have been calculated with the weighted-sum-of-graygas (WSGG) model, and those of the $\mathrm{Al}_{2} \mathrm{O}_{3}$ particles have been solved by the Mie scattering model. The research shows a good similarity between spectral variations of plumes from different scaling solid rockets.

\section{Acknowledgments}

The Guest Editors would like to thank all authors for their contributions to this special issue and the many external reviewers for their excellent work. 


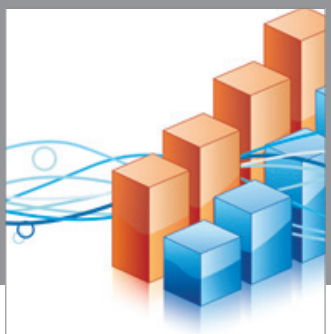

Advances in

Operations Research

vatem alat4

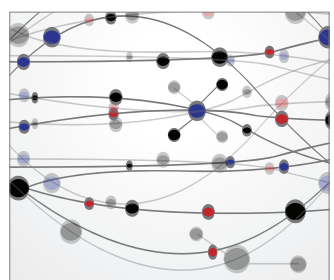

\section{The Scientific} World Journal
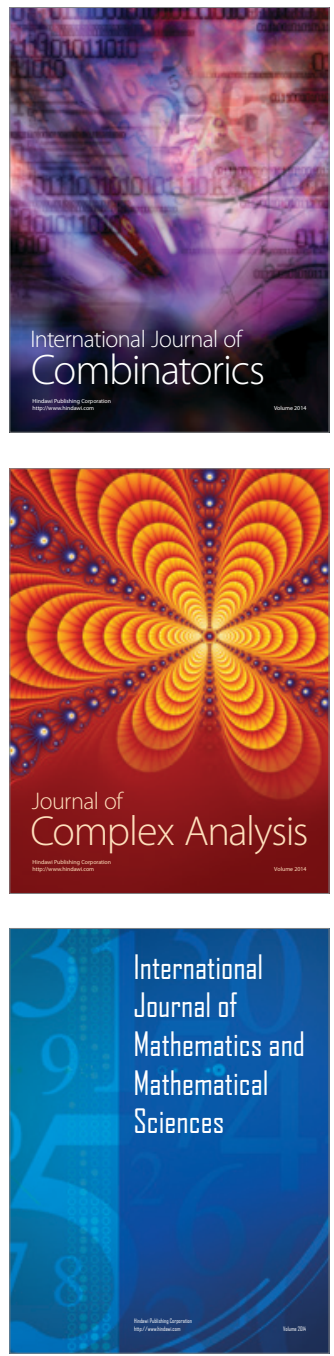
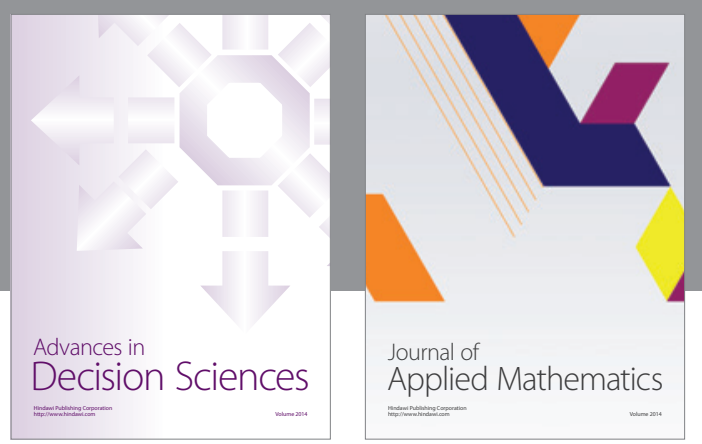

Algebra

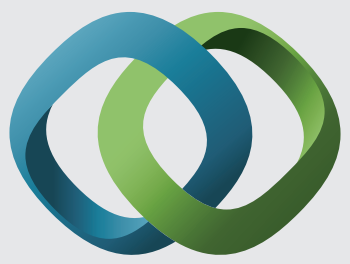

\section{Hindawi}

Submit your manuscripts at

http://www.hindawi.com
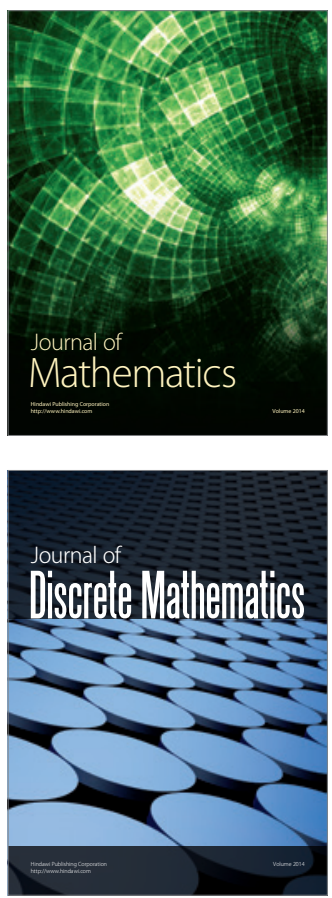

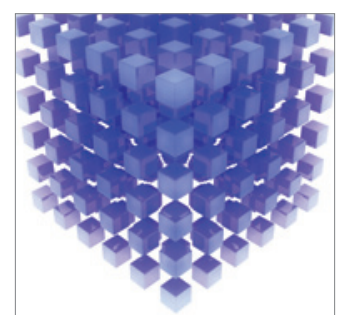

Mathematical Problems in Engineering
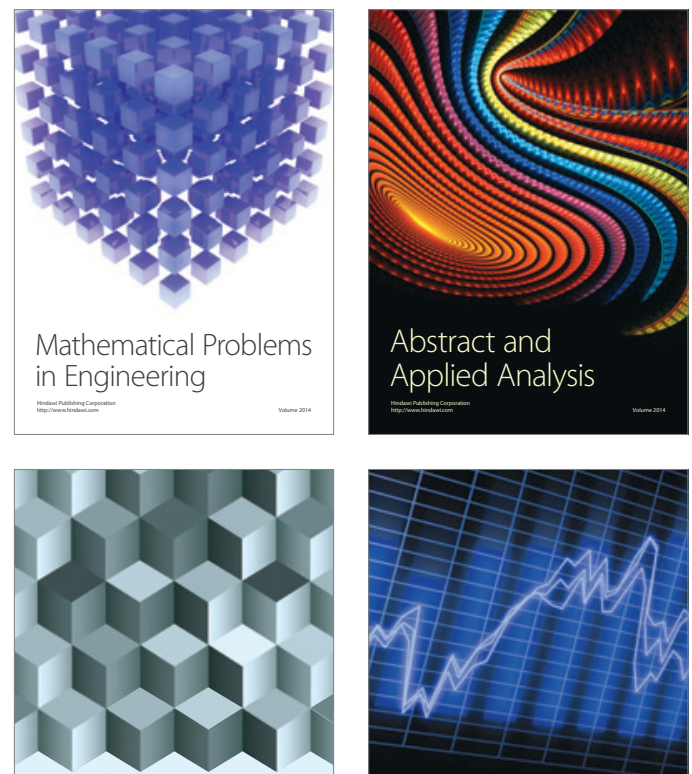

Journal of

Function Spaces

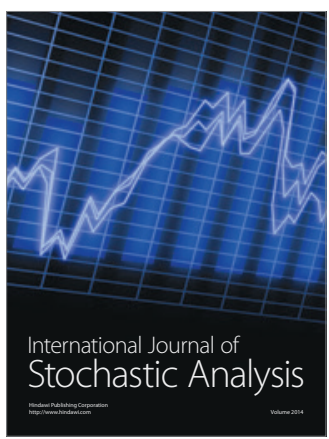

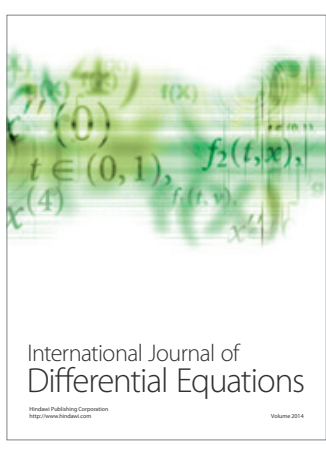
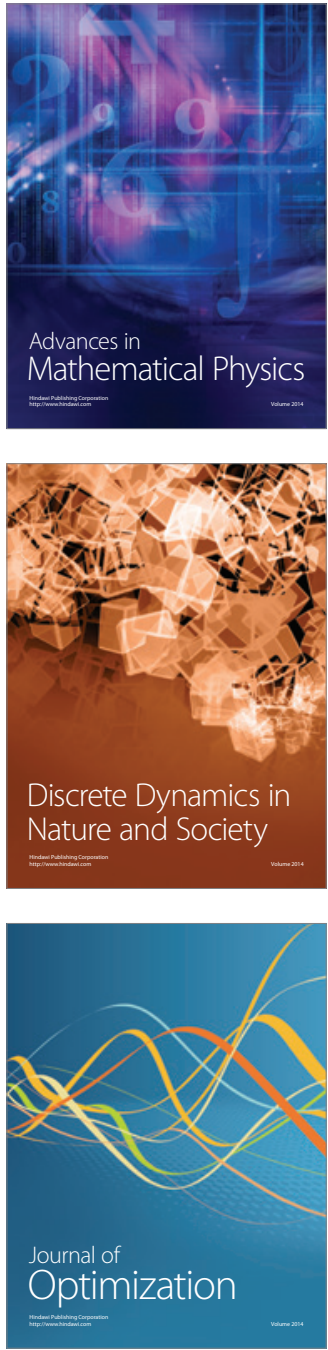